\title{
Beyond fertility preservation: role of the oncofertility unit in the reproductive and gynecological follow-up of young cancer patients
}

\author{
Claudia Massarotti ${ }^{1, *}$, Paola Scaruffi ${ }^{2}$, Matteo Lambertini ${ }^{3,4}$, Fausta \\ Sozzi ${ }^{2}$, Valentino Remorgida', and Paola Anserini ${ }^{2}$
}

\begin{abstract}
'Academic Unit of Obstetrics and Gynecology, DINOGMI Department, University of Genova, 16132, Genova, Italy ${ }^{2}$ Physiopathology of Human Reproduction Unit, IRCCS Ospedale Policlinico San Martino, I6132, Genova, Italy ${ }^{3}$ Department of Medical Oncology, U.O.C. Clinica di Oncologia Medica, IRCCS Ospedale Policlinico San Martino, 16132, Genova, Italy ${ }^{4}$ Department of Internal Medicine and Medical Specialties (DiMI), School of Medicine, University of Genova, 16132, Genova, Italy

*Correspondence address. Physiopathology of Human Reproduction Unit, IRCCS Ospedale Policlinico San Martino, Largo R. Benzi, I0, I6I32 Genova, Italy. Tel: +39-0 10-5555847; Fax: +39-010-5556909; E-mail: claudia.massarotti@gmail.com
\end{abstract}

Submitted on April II, 2019; resubmitted on May 26, 2019; editorial decision on June 3, 2019

STUDY QUESTION: Are there reasons that motivate young cancer survivors to ask for follow-up visits at an oncofertility unit?

SUMMARY ANSWER: Cancer survivors request oncofertility follow-up visits for the management of treatment-related side effects or ovarian reserve evaluation, even if not (or not yet) wishing for a pregnancy.

WHAT IS KNOWN ALREADY: Personalised oncofertility counselling before gonadotoxic therapies is considered standard of care for young women with newly diagnosed cancer. However, the long-term follow-up of these patients in an oncofertility unit is not described in the literature other than for the use of cryopreserved material.

STUDY DESIGN, SIZE, DURATION: We retrospectively examined rates and reasons for the first follow-up visits of I54 consecutive young female cancer patients (age range: 18-40 years) who underwent a pre-treatment consultation between January 2012 and June 2017. Demographic and clinical data were collected, as well as information about the chosen fertility preservation method, if any.

PARTICIPANTS/MATERIALS, SETTING, METHODS: Rates and reasons for follow-up visits were collected and expressed as percentages. Different reasons were examined in the whole cohort and stratified for type of malignancy. Possible predictive factors for return to the follow-up visit (age, nulliparity, presence of a partner, neoplasm, having cryopreserved material) were investigated through logistic regression.

MAIN RESULTS AND THE ROLE OF CHANCE: Out of I54 patients, 74 returned to the oncofertility unit (48.1\%) for a follow-up visit. The first visit was requested mostly at the end of anticancer therapies $(51.3 \%$ versus $40.5 \%$ during therapies and $8.1 \%$ after cancer relapse). Among these patients, only $10.8 \%$ returned for the first time because they were actively desiring a pregnancy. For the others, the most common reasons for consultations were management of gynecological adverse effects of therapies (29.7\%) and evaluation of ovarian reserve not linked to an immediate desire for a pregnancy (39.2\%). Other patients asked for contraception (4.1\%), menopause counselling (5.4\%), or new fertility preservation counselling because of cancer relapse (10.8\%). None of the examined factors were significantly predictive of return to the oncofertility unit.

LIMITATIONS, REASONS FOR CAUTION: These findings represent the experience of a single centre. A longer duration of follow-up would be needed to provide more precise information on this regard.

WIDER IMPLICATION OF THE FINDINGS: The role of an oncofertility unit should not be limited to proposing fertility preservation procedures. In the management of young adult cancer patients, the reproductive medical specialist should be considered a key figure not only before but also during and after anticancer treatments to explore salient aspects of gynecological and reproductive health. 
STUDY FUNDING/COMPETING INTEREST(S): This research did not receive any specific funding. M.L. served as a consultant for Teva and received honoraria from Theramex outside the submitted work. The other authors declare no conflict of interest.

TRIAL REGISTRATION NUMBER: N.A.

Key words: oncofertility / fertility preservation / quality of life after cancer / cancer survivors / pregnancy after cancer / oncofertility unit

\section{Introduction}

Cancer diagnosis in women of reproductive age accounts for 3-10\% of cancer worldwide (Fidler et al., 2017; Siegel et al., 2019). While life expectations for these patients have significantly increased in the past decades, a high proportion of them require therapies that are potentially gonadotoxic (Lambertini et al., 20l6). Cryopreservation techniques developed for infertility treatments have been applied for fertility preservation since the late 1990s and ultimately has led to an increased role of oncofertility units in the management of these patients (Diaz-Gacia et al., 20 I8; Cobo et al., 20 I 8; Gellert et al., 20 I8; Massarotti et al., 20 I7). Several scientific societies have developed specific guidelines focused on fertility preservation to underline the importance of oncofertility counselling in young women who are candidates to potentially gonadotoxic treatments due to a newly diagnosed cancer (Oktay et al., 20 I8; Peccatori et al., 20I3; International Society for Fertility Preservation, 20 12; Ethics Committee of American Society for Reproductive Medicine, 2013; ASRM, 20I3b; Lambertini et al., 20I7a).

While the role of reproductive medical specialists for the management of young cancer patients is well-established at the time of diagnosis, the need for this professional figure during oncological follow-up is less clear for survivors, except for those willing to undergo assisted reproductive technology procedures using their cryopreserved material. The utilisation rate for frozen embryos is reported to be $10-23 \%$ in small cohorts of cancer patients (Barcroft et al., 2013; Dolmans et al., 20I5; Luke et al., 2016), and data about frozen oocytes and ovarian tissue utilisation rates, although still incomplete and inconclusive, are around 5\% (Diaz-Garcia et al., 2018; Martinez et al., 20I4; Drukenmiller et al., 2016). No other reasons for a follow-up at the oncofertility unit are reported and its potential long-term benefits (both on reproductive potential and quality of life) have gained little attention so far.

A recent study has reported that cancer survivors are more likely to use emergency contraception than their peers (Medica et al., 20।8), opening a debate on the best way to empower them with fertility awareness and enhancing the need for a multidisciplinary approach that includes the fertility specialist also during oncologic follow-up (Nahata and Quinn, 2018). Women who are not (or not yet) wishing for a pregnancy could benefit from a long-term follow-up for managing the gynecological adverse effects of anticancer therapies, counselling on contraception or menopause, or evaluation of their post-treatment ovarian reserve and reproductive potential. Moreover, data about the real gonadotoxicity of most therapies, especially the newest targeted agents, and the probability of premature ovarian insufficiency (POI) are still incomplete (Lambertini et al., 20I6). Indeed, not all women who have cryopreserved will need to use their frozen gametes or embryos to obtain a pregnancy, but they could still need the specialist consultation for various reproductive and fertility issues. A proper follow-up at an oncofertility unit would also allow the collection of data about the gonadotoxicity of the new targeted agents, an issue that will become even more important in coming years.
In this context, it is of great relevance to understand what are patients' long-term requests from the reproductive medicine specialists. This study aims at better defining these needs by evaluating the reasons for return to follow-up visits at an oncofertility unit.

\section{Materials and Methods}

\section{Study design and participants}

This is a retrospective analysis of prospectively collected data from oncofertility consultations at a single oncofertility unit. The previous studies using this data received ethical approval (CERLiguria no. 032REG2013). Eligible women for this study were newly diagnosed post-menarche cancer patients under the age of 40 years, who underwent a pre-treatment consultation between January 2012 and June 2017. Since follow-up visits are the main focus of this study, women deceased before having the chance to return were excluded, as were women with severe psychiatric disorders. All patients signed a written agreement form for the use of their data for clinical research.

\section{Oncofertility unit}

This study was conducted in a centre located within an assisted reproduction service, in a gynecological department of a university tertiary hospital. The centre has an active collaboration in terms of patients' referral from both oncologists and hematologists of all cancer services within the same hospital and the survivorship clinic of the nearby pediatric hospital as well as several other smaller regional institutions. Pre-therapy consultations and follow-up visits are performed by gynecologists with expertise in reproductive endocrinology and infertility, specifically trained through internal educational sessions and multidisciplinary discussions of clinical cases (disease management team meetings). Psychological support is available for all patients on request.

\section{Study procedures}

For each patient, demographic and clinical data were recorded at her first access, along with information about ovarian reserve, the type of fertility preservation procedure proposed, and acceptance or reasons for refusal. The possibility of scheduling follow-up visits was offered to all patients, regardless the patient choice on fertility preservation techniques. The women were free to decide when and why to return to the fertility specialist; a fast-track booking system specific for these visits simplified the process. Information regarding the reason for the follow-up visit was also reported.

\section{Study objectives and statistical analysis}

The primary objective was to investigate the reasons for young cancer patients returning to a follow-up visit at an oncofertility unit after a first consultation performed before starting anticancer therapies. In addition, potential predictive factors for return to follow-up were explored. 
Table I Characteristics of study participants: total sample and only patients who returned for a follow-up visit.

\begin{tabular}{|c|c|c|}
\hline & All patients $(n=154) n(\%)$ & $\begin{array}{l}\text { Patients who returned for a } \\
\text { follow-up visit }(n=74) n(\%)\end{array}$ \\
\hline Age at diagnosis, years & $31[26-36]$ & $31[27-35]$ \\
\hline Presence of a partner at diagnosis & $57.7 \%(89 / 154)$ & $68.9 \%(51 / 74)$ \\
\hline Nulliparity at diagnosis & $85.7 \%(132 / 154)$ & $79.8 \%(59 / 74)$ \\
\hline \multicolumn{3}{|l|}{ Type of malignancy } \\
\hline Breast cancer & $47.4 \%(73 / 154)$ & $51.3 \%(38 / 74)$ \\
\hline Hematologic cancer & $31.8 \%(49 / 154)$ & $28.4 \%(21 / 74)$ \\
\hline Others & $20.8 \%(33 / 154)$ & $20.3 \%(15 / 74)$ \\
\hline High gonadotoxic therapy & $22.1 \%(34 / 154)$ & $22.9 \%(17 / 74)$ \\
\hline \multicolumn{3}{|l|}{ Ovarian reserve at diagnosis } \\
\hline Anti-Mullerian hormone-AMH (ng/ml) & $1.77[1.06-3.29]$ & $1.77[1.16-3.66]$ \\
\hline Antral follicular count-AFC ( $n$ ) & $12[8-18.5]$ & $12[8-17]$ \\
\hline \multicolumn{3}{|l|}{ Fertility preservation procedures * } \\
\hline Oocyte cryopreservation * & $22.7 \%(35 / 154)$ & $25.7 \%(19 / 74)$ \\
\hline Ovarian tissue cryopreservation * & $11.7 \%(18 / 154)$ & $12.2 \%(9 / 74)$ \\
\hline Ovarian transposition * & $5.8 \%(9 / 154)$ & $4.05 \%(3 / 74)$ \\
\hline Cryopreservation procedures not proposed & $25.3 \%(39 / 154)$ & $20.2 \%(15 / 74)$ \\
\hline GnRH agonist ** & $66.8 \%(103 / 154)$ & $67.6 \%(50 / 74)$ \\
\hline
\end{tabular}

Continuous data are expressed as median with first and third quartiles, and categorical data are expressed as absolute number and percentage. Reasons for the first follow-up visit among subgroups of patients affected by different malignancies were compared through chi square test. Logistic regression was used to explore the presence of potential predictive factors for return to follow-up. A $P$-value $<0.05$ was considered statistically significant. Statistical analysis was carried out with the software R, version 3.5.2.

\section{Results}

\section{Characteristics of study participants}

We enrolled in the study a total of I54 women. Demographic and clinical characteristics of the patients are reported in Table I. The two most common malignancies were breast $(n=73,47.4 \%)$ and hematological ( $n=49,31.8 \%$ ) cancers. Half of the patients $(n=89$, $57.7 \%$ ) reported to be in a stable relationship at the time of the first visit and the majority of them $(n=132,85.7 \%)$ were childless. On note, only a minority $(n=34,22.1 \%)$ received treatments with proven high risk of gonadotoxicity (i.e. conditioning for bone marrow transplantation). The other patients underwent therapies with low-tomoderate or unknown gonadotoxicity risk.

\section{Cryopreservation procedures}

In total, 57 patients (37\% of the total cohort) underwent a fertility preservation procedure. Oocyte cryopreservation was performed for 35 patients $(22.7 \%)$, ovarian tissue cryopreservation for 18 patients
( 1 I.7\%), and ovarian transposition for 9 patients (5.8\%). Five patients did more than one procedure. A total of 103 patients (66.8\%) used monthly injection of gonadotropin-releasing hormone $(\mathrm{GnRH})$ analogs during chemotherapy for gonadal protection. Three patients who refused a cryopreservation procedure before a low gonadotoxicity first-line therapy underwent ovarian tissue cryopreservation before a second-line therapy of higher gonadotoxic potential.

Of the total sample, 39 women (25.3\%) did not undergo any fertility preservation procedures for medical reasons. Of the remaining II5 patients, 6I (53\%) refused any cryopreservation procedure as a personal choice. Patients flow and uptake of fertility preservation procedures, including reasons for non-eligibility and refusal, are shown in Fig. I.

\section{Return to the oncofertility unit for a follow-up visit}

Almost half of the total cohort of patients $(n=74,48 \%)$ returned at least once to the oncofertility unit for a follow-up visit.

Half of the patients returned to the oncofertility unit for the first time after a mean time of 3.92 months ( \pm 2.44 months) following the end of the anticancer therapies (51.3\%), 40.5\% returned during treatment, and $8.1 \%$ returned after having experienced a relapse.

Regarding the reasons for follow-up visits, only eight patients ( $10.8 \%)$ returned actively desiring a pregnancy; among these, three had a spontaneous pregnancy, four started an ovarian stimulation for ART, and one used her oocytes cryopreserved at the time of diagnosis.

The main reasons for the first visit were evaluation of the gonadotoxic effects on the reproductive potential (39.2\%) and management 


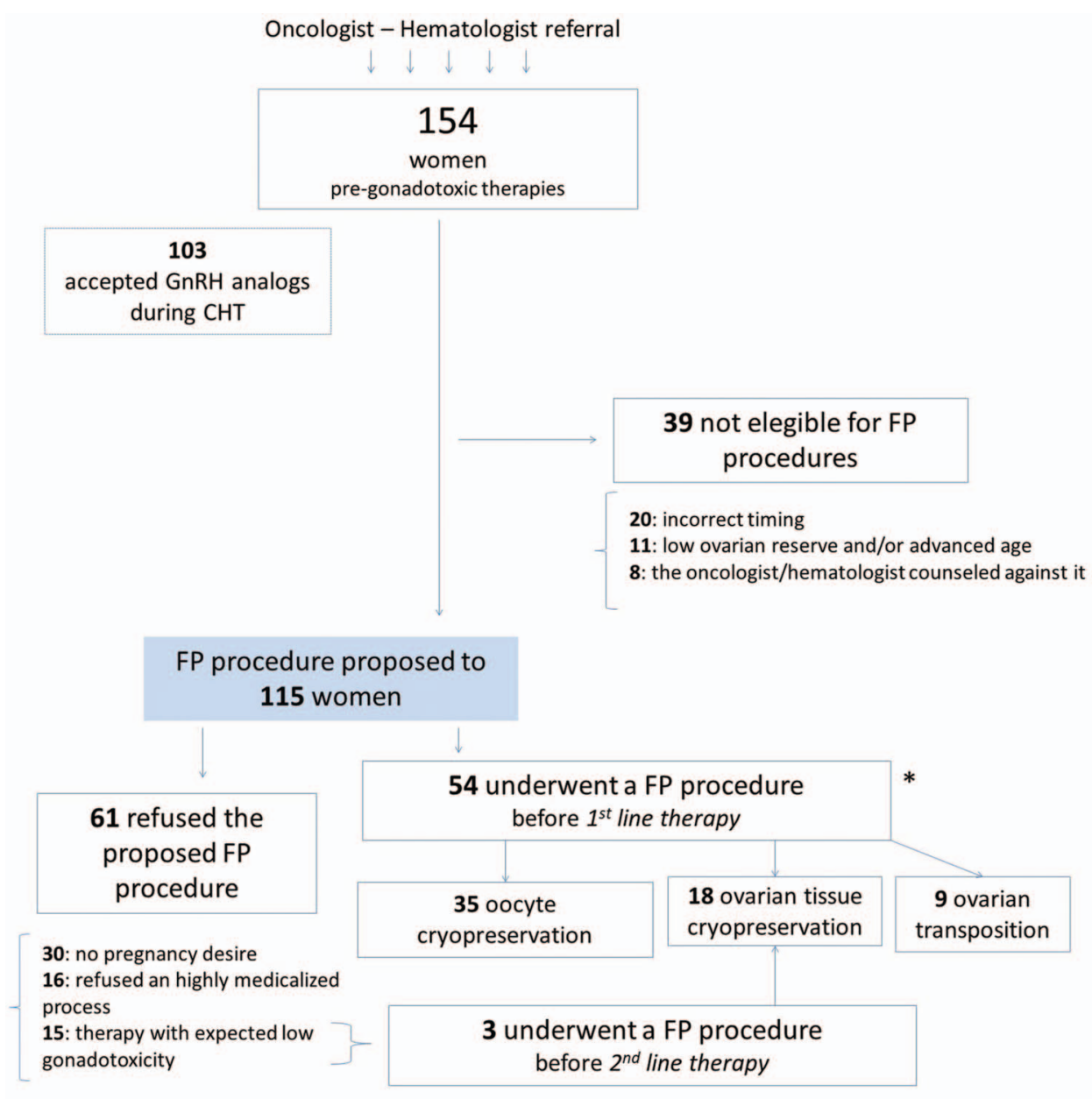

* 5 patients did more than one procedure

Figure I Patients flow and uptake of fertility preservation procedures.

$\mathrm{FP}=$ fertility preservation; $\mathrm{GnRH}$ analogs = gonadotropin-releasing hormone analogs; $\mathrm{CHT}=$ chemotherapy.

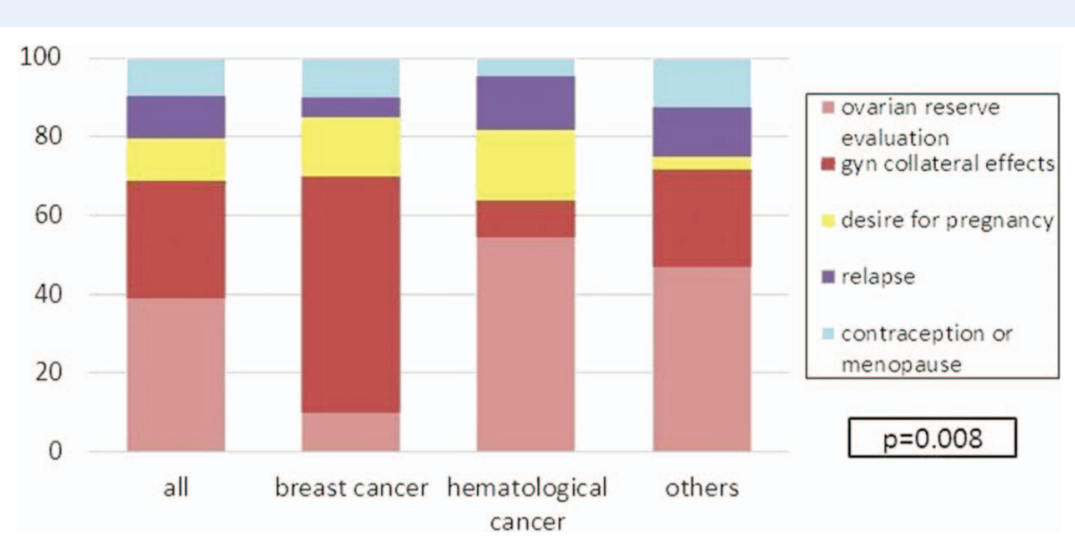

Figure 2 Reason for a follow-up visit, overall and according to type of malignancy.

Data is expressed as percentage and compared with chi square test. Statistically significant difference $(P=0.00 \mathrm{I})$. gyn $=$ gynaecological. 
Table 2 Predictive factors of return to the oncofertility unit for a follow-up visit.

\begin{tabular}{|c|c|c|}
\hline Potential predictive factor & $\begin{array}{l}\text { Odds ratio } \\
\text { [95\% C.I.] }\end{array}$ & $P$-value \\
\hline Being in a relationship at diagnosis & $2.29[0.94-4.65]$ & NS \\
\hline Being childless at diagnosis & $0.61[0.21-1.76]$ & NS \\
\hline Pre-treatment $\mathrm{AMH}<\mathrm{I} \mathrm{ng} / \mathrm{ml}$ & $1.19[0.49-2.91]$ & NS \\
\hline Breast or hematological cancer & $1.53[0.66-3.54]$ & NS \\
\hline High gonadotoxicity of therapies & $1.22[0.53-2-80]$ & NS \\
\hline $\begin{array}{l}\text { Prior access to a cryopreservation } \\
\text { procedure }\end{array}$ & $1.13[0.55-2.30]$ & NS \\
\hline
\end{tabular}

of treatment-related gynecological adverse effects (29.7\%). Patients required consultations also for counselling about contraception (4.1\%) and menopausal symptoms (5.4\%). Moreover, eight patients ( $10.8 \%)$ returned before starting a new and more gonadotoxic therapy for a relapse of their malignancy.

Figure 2 shows the reasons for follow-up visits according to type of malignancy (breast, hematological, others). The most common reason for follow-up was management of gynecological adverse effects for breast cancer patients and evaluation of reproductive potential for women with hematological malignancies.

Table 2 examines possible predictive factors of return to the oncofertility unit for a follow-up visit. Being childless, having a low ovarian reserve at diagnosis, exposure to high gonadotoxic therapies, type of malignancy, and a prior cryopreservation procedure were not shown to be predictors of return to a follow-up visit.

\section{Discussion}

While the role of the oncofertility unit in counselling on infertility risk and fertility preservation options is universally endorsed (Oktay et al., 20 I8; Peccatori et al., 20 I3; International Society for Fertility Preservation, 2012; Ethics Committee of American Society for Reproductive Medicine, 20 I3; ASRM, 20I3b; Lambertini et al., 20 I7a), less attention is paid to its function in cancer survivors (Nahata and Quinn, 20I8; Anazodo et al., 20 I9), except when patients require the access to ART procedures and the use of cryopreserved material. To our knowledge, this is the first study reporting rates of and reasons for oncofertility follow-up in young cancer patients, with the aim to better define a potential broader role of the oncofertility unit in their management.

For young cancer patients, fertility is undoubtedly a relevant issue with the majority of them showing concerns about the potential risk of treatment-related $\mathrm{POI}$ and infertility after appropriate oncofertility counselling (Lambertini et al., 20 I8; Ruddy et al., 20I4). Undoubtedly, the timely communication between oncologists, hematologists, and reproductive medical specialists enables a personalised counselling in which ovarian reserve can be evaluated, the gonadotoxicity of therapies can be discussed, and a fertility preservation procedure can be proposed whenever appropriate (Vu et al., 2017; Von Wolff et al., 20I5). In our experience, oncologists and hematologists give general information about gonadotoxic risks of treatments and the possibility of undergoing fertility preservation procedures, proposing a dedicated oncofertility consultation with the reproductive medical specialists to all women who desire more information regardless of their attitude towards cryopreservation procedures. The acceptance rate of cryopreservation procedures in our study seems low (49.6\%); however, notably, the majority of these patients chose not to undergo a procedure as a conscious personal decision after complete counselling (either because they completed their family planning or due to the low gonadotoxicity of the proposed therapies). Nevertheless, also patients who have completed their family planning and have no desire for other children may be worried about the adverse effects associated with POI development and desire reliable information about their ovarian reserve (Vu et al., 20 I7; Lambertini et al., 20 I8). It is plausible that this interest remains strong also during and after the end of anticancer therapies. As suggested by our results, the ideal path of a young woman at the oncofertility unit starts at cancer diagnosis but continues during and after gonadotoxic therapies, and this is not only limited to pregnancy desire or use of cryopreserved material (Fig. 3).

Various evidence in the literature shows how the needs of cancer survivors may be not yet met by gynecologists in an optimal way over the long term (Schover, 2018; Anderson et al., 2018). Reduced sexual quality of life (Schover, 20I8) and reduced chance of pregnancy (Anderson et al., 2018) were observed among patients with all cancer types, including those who underwent surgery without gonadotoxic therapies. Gynecological adverse effects of therapies and treatmentrelated loss of fertility clearly play a relevant role in these results, but other psychological and social factors (i.e. concern about recurrence, reproductive issues not adequately addressed, or other health issues) may contribute (Howard-Anderson et al., 20 I2; Schmidt et al., 20I6).

During gonadotoxic therapies, early gynecological adverse effects are a significant issue. In the literature, cancer patients, especially those treated with chemotherapy, report low rates of overall sexual satisfaction and less arousal and pleasure, and these problems are not always adequately addressed (Condorelli et al., 2019; Schover, 20I8; Dominick et al., 20।5). This requires specific expertise, especially in women who had hormone sensitive cancers and, therefore, limited treatment options (American College of Obstetricians and Gynecologists' Committee on Gynecologic Practice and Farrell, 20 I6). Coherently, $29.7 \%$ of patients returned to have a follow-up visit for treatment-related issues (i.e. vaginal dryness or other menopausal symptoms). This was the main reason for the return of women with breast cancer, who are usually subjected to treatments of low-tomoderate gonadotoxicity risk, followed by 5 to 10 years of adjuvant endocrine therapy for hormone-sensitive cancers, which is associated with significant side effects.

Deeply linked to this issue, and pivotal in the restoration of a satisfactory sexual life, patients need a safe and reliable contraception. Indeed, in the literature, data suggest that they are less likely to receive adequate contraception counselling, with rates up to $56 \%$ of survivors reporting no family planning counselling at all (CastroSanchez et al., 2018). Additionally, they are less likely to be satisfied and compliant with the prescribed method (Blouet et al., 2019) and more likely to wrongly assume they are infertile and to face unwanted pregnancies (Medica et al., 2018; Hadnott et al., 2019). Information on contraception during chemotherapy, along with information on fertility preservation, is part of our pre-therapy counselling. Usually, oral combined contraceptives or a vaginal ring is suggested to patients without hormone-sensitive malignancies; in all other cases, barrier 


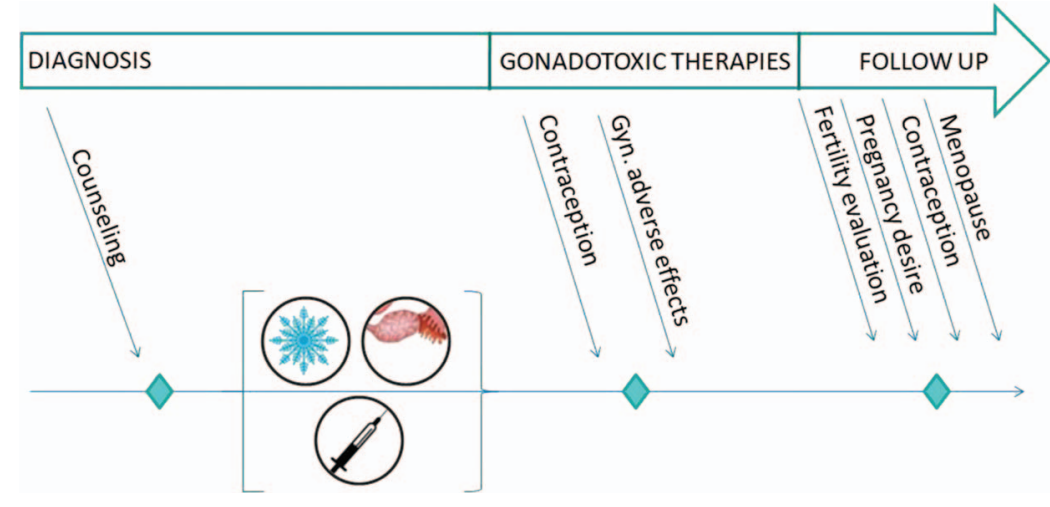

Figure 3 Proposed path of young cancer patients at an oncofertility unit. gyn = gynaecological.

contraception or copper intra-uterine device is proposed. In this study, we reported that $4.1 \%$ of visits are exclusively for contraception counselling; this seems low, but, since we discuss the topic at every visit, this percentage represents only the patients who are not satisfied with the current method and who have requested an additional consultation to re-discuss it. More data are needed to improve the counselling of young cancer patients on this regard.

An oncofertility unit, in our model, should manage patients over the long term, providing education to raise fertility awareness, to obtain the best possible sexual quality of life, to discuss the timing of a potential pregnancy and best pathway to achieve it, or to provide reliable contraception and gynecological care (including preventive measures). Also in women who develop POI, the role of the reproductive specialist is crucial for the management of hormonal therapy, if not contraindicated, or specific therapies for specific adverse effects (e.g. sexual health, bone health) (in collaboration with endocrinologists) and cardiovascular evaluation (with cardiologists). Indeed, a long-term model of care enables a punctual and dynamic evaluation of reproductive potential and gonadotoxic damage of treatments at multiple time points, with the double aim of empowering the patient with reliable information on her ovarian reserve and to better understand the real damage to fertility of anticancer therapies, on which we still have little information, especially for the new targeted agents (Lambertini et al., 2016). Surveys among gynecologists without specific expertise in reproductive endocrinology and infertility show a generally low level of specific knowledge and sometimes even the inability to correctly interpret and contextualise hormonal levels assays in an infertile woman (Revelli et al., 20I5). Fertility evaluation post gonadotoxic therapies is even harder for many reasons. First of all, traditional markers may be less useful after prior exposure to gonadotoxic therapies. In fact, antiMullerian hormone (AMH) levels are reported to be particularly low right after chemotherapy, also in women with residual fertility (Freour et al., 2017; Anderson et al., 2017). In addition, a regular menstrual cycle is not always an indicator of fertility restoration while, on the other hand, amenorrhea could be only temporary (Partridge et al., 2010; Decanter et al., 2018).

The majority of women in our cohort returned for the first follow-up visit for a reproductive potential evaluation (39.2\%), and this included patients who opted out of fertility preservation techniques when offered, proving how the interest remains strong also after treatments. This was the main reason for follow-up in women with hematological malignancies, who are subjected to therapies that span from low gonadotoxicity (i.e. first line therapy for lymphoma with $A B V D$ (adriamycin/bleomycin/vinblastine/dacarbazine) regimen protocol) to high gonadotoxicity (i.e. conditioning for bone marrow transplantation). Hence, both patients who chose not to undergo a cryopreservation procedure because of the low gonadotoxicity of the therapies and patients who underwent high gonadotoxicity therapies (independently of whether or not having had a cryopreservation procedure) are both expected to desire a reproductive potential evaluation. There is no consensus on when and how fertility investigation after cancer therapy is predictive of future fertility. We usually perform ovarian reserve evaluation at each visit (i.e. $\mathrm{AMH}$ levels, antral follicular count, presence of menses), informing the patient that their results, even hypergonadotropic amenorrhea, are not conclusive of residual fertility. In our opinion, since ovarian reserve evaluation cannot be done in a single visit and most of the markers are dynamic and vary during and after the end of gonadotoxic therapies, a proper follow-up at the oncofertility unit is the key for both patient's empowerment and advancement in scientific knowledge.

In patients who desire a pregnancy, fertility awareness enables them to better define when and how to safely attempt a pregnancy after cancer. The likelihood of pregnancy in women who previously had a malignancy is reduced by $\sim 38 \%$ compared to the general population (Anderson et al., 2018), in all age groups at diagnosis and in all malignancies. The advent of new therapies and the consequently reduced morbidity and mortality, as well fertility preservation options, has increase the incidence of pregnancy after cancer in the last 10 years (Anderson et al., 20l8), but there is still a long way to go. The collaboration between woman, reproductive medical specialist, and oncologist or hematologist can minimise the risks and maximise the chances of success, since there is actually no perfect time to try for a pregnancy after cancer (Lambertini et al., 20l7b), and only the combination of data about the malignancy and the woman-specific reproductive potential can lead to a pregnancy attempt, which is both safe and effective. Thanks to the information acquired with a long-term model of care, the patient can be adequately counselled on the best way to achieve a pregnancy (i.e. trying to conceive spontaneously, using 
reproductive technologies or her cryopreserved material, or using an oocyte donor and/or a gestational carrier). In our cohort, only $10.8 \%$ of women returned to follow-up for the first time actively desiring a pregnancy. Many other, included the ones who already returned for other reasons, may try to conceive in the future, since our follow-up is relatively short.

In conclusion, our experience suggests the needs of cancer patients in accessing the oncofertility unit for counselling and care even when they do not want (or cannot yet have) a pregnancy. Fertility and gynecological health care after cancer are a result of many complex variables and require tailored and long-term evaluation. Continuity of care, as requested by patients in our experience, could ultimately increase the reproductive potential, empowering women to make conscious choices on when and how to attempt a pregnancy, if desired, but also on effective contraception and, in general, on improving their quality of life. Although our sample is small and reflects the experience of a single centre, it contributes in describing the heterogeneous needs of young cancer patients before, during and after gonadotoxic therapies, advocating for a broader role of an oncofertility unit. With the advancement of research in this setting, the role of the oncofertility unit is destined to change from acute 'rescue therapy' to long-term counselling and care that can and must be offered, including to women for whom a fertility preservation procedure is not indicated or not requested.

\section{Authors' roles}

C.M. contributed to the study design and data collection, performed the statistical analysis, and drafted the manuscript. P.S. and M.L. contributed to the study design and patient management and critically revised the article. F.S. contributed to the clinical management of patients and data collection. V.R. critically revised the study protocol and the manuscript. P.A. designed the study, was responsible of clinical management of patients and general supervision, and critically revised the article. All authors read, revised, and approved the final version of the manuscript.

\section{Funding}

This research did not receive any specific grant from funding agencies in the public, commercial, or not-for-profit sectors.

\section{Conflict of interest}

M.L. served as a consultant for Teva and received honoraria from Theramex outside the submitted work. The other authors declare no conflict of interest.

\section{References}

American College of Obstetricians and Gynecologists' Committee on Gynecologic Practice, Farrell R. ACOG Committee opinion no. 659: the use of vaginal estrogen in women with a history of estrogendependent breast cancer. Obstet Gynecol 2016; 1 27:e93-e96.
Anazodo A, Laws P, Logan S, Saunders C, Travaglia J, Gerstl B, Bradford N, Cohn R, Birdsall M, Barr R et al. How can we improve oncofertility care for patients? A systematic scoping review of current international practice and models of care. Hum Reprod Update 2019;25:159-179.

Anderson RA, Brewster DH, Wood R, Nowell S, Fischbacher C, Kelsey TW, Wallace WHB. The impact of cancer on subsequent chance of pregnancy: a population-based analysis. Hum Reprod 2018;33: $1281-1290$.

Anderson RA, Mansi J, Coleman RE, Adamson DJA, Leonard RCF. The utility of anti-Müllerian hormone in the diagnosis and prediction of loss of ovarian function following chemotherapy for early breast cancer. Eur J Cancer 2017;87:58-64.

Barcroft J, Dayoub N, Thong KJ. Fifteen year follow-up of embryos cryopreserved in cancer patients for fertility preservation. J Assist Reprod Genet 2013;30: |407-1413.

Blouet A, Zinger M, Capitain O, Landry S, Bourgeois H, Seegers VT, Pointreau $Y$. Sexual quality of life evaluation after treatment among women with breast cancer under 35 years old. Support Care Cancer 2019;27:879-885.

Castro-Sanchez A, Martinez-Cannon BA, Platas A, Mohar A, Fonseca A, Vega Y, Fimbres-Morales A, Villarreal-Garza C. Suboptimal use of effective contraceptive methods in young Mexican women with breast cancer. J Global Oncol 2018;4:1-7.

Cobo A, García-Velasco J, Domingo J, Pellicer A, Remohí J. Elective and onco-fertility preservation: factors related to IVF outcomes. Hum Reprod 2018;33:2222-2231.

Condorelli M, Lambertini M, Del Mastro L, Boccardo F, Demeestere I, Bober SL. Fertility, sexuality and cancer in young adult women. Curr Opin Oncol 2019;15.

Decanter C, Cloquet M, Dassonneville A, D’Orazio E, Mailliez A, Pigny $P$. Different patterns of ovarian recovery after cancer treatment suggest various individual ovarian susceptibilities to chemotherapy. Reprod Biomed Online 2018;36:71 I-7I8.

Diaz-Garcia C, Domingo J, Garcia-Velasco JA, Herraiz S, Mirabet V, Iniesta I, Cobo A, Remohí J, Pellicer A. Oocyte vitrification versus ovarian cortex transplantation in fertility preservation for adult women undergoing gonadotoxic treatments: a prospective cohort study. Fertil Steril 20 I 8; 1 09:478-485.

Dolmans MM, Hollanders de Ouderaen S, Demylle D, Pirard C. Utilization rates and results of long-term embryo cryopreservation before gonadotoxic treatment. J Assist Reprod Genet 2015;32: 1233-1237.

Dominick SA, McLean MR, Whitcomb BW, Gorman JR, Mersereau JE, Bouknight JM, Su HI. Contraceptive practices among female cancer survivors of reproductive age. Obstet Gynecol 2015; I 26: 498-507.

Drukenmiller S, Goldman KN, Labella PA, Fino ME, Bazzocchi A, Noyes $N$. Successful oocyte cryopreservation in reproductive-aged cancer survivors. Obstet Gynecol 2016; 1 27:474-480.

Ethics Committee of American Society for Reproductive Medicine. Fertility preservation and reproduction in patients facing gonadotoxic therapies: a committee opinion. Fertil Steril 2013; 1 00: | 224-1231.

Fidler MM, Gupta S, Soerjomataram I, Ferlay J, Steliarova-Foucher E, Bray F. Cancer incidence and mortality among young adults aged 20 39 years worldwide in 2012: a population-based study. Lancet Oncol 2017; 18:1579-1589. 
Fréour T, Barrière $P$, Masson D. Anti-müllerian hormone levels and evolution in women of reproductive age with breast cancer treated with chemotherapy. Eur J Cancer 2017;74: I-8.

Gellert SE, Pors SE, Kristensen SG, Bay-Bjørn AM, Ernst E, Yding AC. Transplantation of frozen-thawed ovarian tissue: an update on worldwide activity published in peer-reviewed papers and on the Danish cohort. J Assist Reprod Genet 2018;35:56I-570.

Hadnott TN, Stark SS, Medica A, Dietz AC, Martinez ME, Whitcomb BW, Su HI. Perceived infertility and contraceptive use in the female reproductive-age cancer survivor. Fertil Steril 20 19; I I I:763-770.

Howard-Anderson J, Ganz PA, Bower JE, Stanton AL. Quality of life, fertility concerns, and behavioral health outcomes in younger breast cancer survivors: a systematic review. I Natl Cancer Inst 2012; 104:386-405.

International Society for Fertility Preservation. Recommendations for fertility preservation in patients with lymphoma, leukemia, and breast cancer. J Assist Reprod Genet 2012;29:465-468.

Jemal A. Cancer statistics, 2019. CA Cancer J Clin 2019;69:7-34.

Lambertini M, Del Mastro L, Pescio MC, Andersen CY, Azim HA Jr, Peccatori F, Costa M, Revelli A, Salvagno F, Gennari A et al. Cancer and fertility preservation: international recommendations from an expert meeting. BMC Med 2016; I 4: I.

Lambertini M, Cinquini M, Moschetti I, Peccatori FA, Anserini P, Valenzano Menada M, Tomirotti M, Del Mastro L. Temporary ovarian suppression during chemotherapy to preserve ovarian function and fertility in breast cancer patients: a GRADE approach for evidence evaluation and recommendations by the Italian Association of Medical Oncology. Eur J Cancer 20 I7a;7 I:25-33.

Lambertini M, Goldrat O, Clatot F, Demeestere I, Awada A. Controversies about fertility and pregnancy issues in young breast cancer patients: current state of the art. Curr Opin Oncol 2017b;29: 243-252.

Lambertini M, Fontana V, Massarotti C, Poggio F, Dellepiane C, lacono G, Abate A, Miglietta L, Ferreccio C, Pescio MC et al. Prospective study to optimize care and improve knowledge on preservation of ovarian function and fertility in young breast cancer patients: first results of the PREgnacy and FERtilty (PREFER) study. The Breast 2018;4I:5।-56.

Luke B, Brown MB, Spector LG, Stern JE, Smith YR, Williams M, Koch L, Schymura MJ. Embryo banking among women diagnosed with cancer: a pilot population-based study in New York, Texas, and Illinois. J Assist Reprod Genet 2016;33:667-674.

Martinez M, Rabadan S, Domingo J, Cobo A, Pellicer A, Garcia-Velasco JA. Obstetric outcome after oocyte vitrification and warming for fertility preservation in women with cancer. Reprod Biomed Online 2014;29:722-728.

Massarotti C, Scaruffi P, Lambertini M, Remorgida V, Del Mastro L, Anserini P. State of the art on oocyte cryopreservation in female cancer patients: a critical review of the literature. Cancer Treat Rev 2017;57:50-57.

Medica ACO, Stark SS, Hadnott TN, Dietz AC, Romero SAD, Natarajan L, Martinez E, Whitcomb BW, Su HI. Use of emergency contraception among female young adult cancer survivors. Fertil Steril 2018;109: I |14-1120.

Nahata L, Quinn GP. Contraception and reproductive health counseling in cancer survivorship -plan a rather than plan B. Fertil Steril 2018;109:1016-1017.

Oktay K, Harvey BE, Partridge AH, Quinn GP, Reinecke J, Taylor HS, Wallace WH, Wang ET, Loren AW. Fertility preservation in patients with cancer: ASCO clinical practice guideline update. J Clin Oncol 2018;36:1994-200I.

Partridge AH, Ruddy KJ, Gelber S, Schapira L, Abusief M, Meyer $M$, Ginsburg E. Ovarian reserve in women who remain premenopausal after chemotherapy for early stage breast cancer. Fertil Steril 2010;94:638-644.

Peccatori FA, Azim HA Jr, Orecchia R, Hoekstra HJ, Pavlidis N, Kesic V, Pentheroudakis G, ESMO Guidelines Working Group. Cancer, pregnancy and fertility: ESMO clinical practice guidelines for diagnosis, treatment and follow-up. Ann Oncol 2013;24:160-170.

Practice Committee of the American Society for Reproductive Medicine. Fertility preservation in patients undergoing gonadotoxic therapy or gonadectomy: a committee opinion. Fertil Steril 20 I 3; 100 : 1214-1223.

Revelli A, Razzano A, Delle Piane L, Casano S, Benedetto C. Awareness of the effects of postponing motherhood among hospital gynecologists: is their knowledge sufficient to offer appropriate help to patients? J Assist Reprod Genet 2016;33:215-220.

Ruddy KJ, Gelber SI, Tamimi RM, Ginsburg ES, Schapira L, Come SE, Borges VF, Meyer ME, Partridge AH. Prospective study of fertility concerns and preservation strategies in young women with breast cancer. J Clin Oncol 20 |4;32: I I I - I I 56.

Schmidt R, Richter D, Sender A, Geue K. Motivations for having children after cancer-a systematic review of the literature. Eur $J$ Cancer Care (Engl) 2016;25:6-17.

Schover LR. Sexual quality of life in men and women after cancer. Climacteric 2018;3 I:I-5.

Siegel RL, Miller KD, Jemal A. Cancer statistics. CA Cancer J Clin 2019;69:7-34.

Von Wolff M, Dittrich R, Liebenthron J, Nawroth F, Shuring AN, Bruckner T, Germeyer A. Fertility preservation counselling and treatment for medical reasons: data from a multinational network of over 5000 women. Reprod Biomed Online 2015;31:605-6I2.

Vu JV, Llarena NC, Estevez SL, Moravek MB, Jeruss JS. Oncofertility program implementation increases access to fertility preservation options and assisted reproductive procedures for breast cancer patients. J Surg Oncol 2017; I I 5: | |6-121. 